\title{
Decoding Ventromedial Hypothalamic Neural Activity during Male Mouse Aggression
}

\author{
Annegret L. Falkner, ${ }^{1}$ Piotr Dollar, ${ }^{4}$ Pietro Perona, ${ }^{4}$ David J. Anderson, ${ }^{2,3}$ and Dayu Lin ${ }^{1}$ \\ ${ }^{1}$ Department of Psychiatry, Institute of Neuroscience, New York University School of Medicine, New York, New York 10016, and ${ }^{2}$ Division of Biology, \\ ${ }^{3}$ Howard Hughes Medical Institute, and ${ }^{4}$ Computation and Neural Systems, California Institute of Technology, Pasadena, California 91125
}

\begin{abstract}
The ventromedial hypothalamus, ventrolateral area (VMHvl) was identified recently as a critical locus for inter-male aggression. Optogenetic stimulation of VMHvl in male mice evokes attack toward conspecifics and inactivation of the region inhibits natural aggression, yet very little is known about its underlying neural activity. To understand its role in promoting aggression, we recorded and analyzed neural activity in the VMHvl in response to a wide range of social and nonsocial stimuli. Although response profiles of VMHvl neurons are complex and heterogeneous, we identified a subpopulation of neurons that respond maximally during investigation and attack of male conspecific mice and during investigation of a source of male mouse urine. These "male responsive" neurons in the VMHvl are tuned to both the inter-male distance and the animal's velocity during attack. Additionally, VMHvl activity predicts several parameters of future aggressive action, including the latency and duration of the next attack. Linear regression analysis further demonstrates that aggressionspecific parameters, such as distance, movement velocity, and attack latency, can model ongoing VMHvl activity fluctuation during inter-male encounters. These results represent the first effort to understand the hypothalamic neural activity during social behaviors using quantitative tools and suggest an important role for the VMHvl in encoding movement, sensory, and motivation-related signals.
\end{abstract}

Key words: aggression; hypothalamus; motivation; physiology

\section{Introduction}

A longstanding yet central question in neuroscience is how instinctive social behaviors, such as aggression, are generated (Tinbergen, 1951). The hypothalamus, a highly conserved limbic structure, has long been known to be involved in aggression, although its specific role in promoting these actions is still poorly understood. Early experiments performed on cats during the 1920s, which severed the hypothalamus from midbrain and brainstem structures, first demonstrated that the intact hypothalamus is necessary for the expression of rage (Bard, 1928). Later, a series of classic electric stimulation experiments showed that complex aggressive actions could be evoked by stimulating the hypothalamus in a variety of species (Putkonen, 1966; Lipp and

Received Dec. 6, 2013; revised March 20, 2014; accepted March 21, 2014.

Author contributions: D.J.A. and D.L. designed research; D.L. performed research; P.D. and P.P. contributed unpublished reagents/analytic tools; A.L.F. analyzed data; A.L.F. and D.L. wrote the paper.

This work was supported by the Jane Coffin Child Foundation (D.L.), National Institutes of Health Grant 1R01MH101377-01 (D.L.), Whitehall foundation fellowship (D.L.), the Weston-Havens Foundation (D.J.A.), MH0750053 (D.J.A.), MH085082 (D.J.A.), Ellison Medical Foundation (D.J.A.), Paul G. Allen Family Foundation (D.J.A.), Howard Hughes Medical Institute (D.J.A.), Gordon and Betty Moore Foundation (P.P. and D.J.A.), and ONR MURI Grant \#N0014-10-1-0933 (P.P.). We thank Alex Ramirez for helpful conversations, Mickey Goldberg, Li Chin Wong, and Susanne Tranguch for editorial comments, Ciocchi Stephane for help in electrode construction, and Robert Robbins for video annotation.

The authors declare no competing financial interests.

Correspondence should be addressed to any of the following: Annegret Falkner, Department of Psychiatry, Smilow Neuroscience Institute, New York University School of Medicine, New York, NY 10016, E-mail: annegret.falkner@nyumc.org; David J. Anderson, Division of Biology, California Institute of Technology, Pasadena, CA 91125, E-mail: wuwei@caltech.edu; or Dayu Lin, Department of Psychiatry, Smilow Neuroscience Institute, New York University School of Medicine, New York, NY 10016, E-mail: dayu.lin@nyumc.org.

DOI:10.1523/JNEUROSCI.5109-13.2014

Copyright $\odot 2014$ the authors $\quad 0270-6474 / 14 / 345971-14 \$ 15.00 / 0$
Hunsperger, 1978; Kruk et al., 1983; Siegel and Pott, 1988; Ettrup et al., 2012). In particular, Kruk et al. (1983) mapped out a distributed "hypothalamic attack area" in rats by comparing the effects of hundreds of electrode stimulation sites. Our recent studies in mice demonstrated that optogenetic stimulation of the ventromedial hypothalamus, ventrolateral area (VMHvl), but not its surrounding regions, promotes attack toward males as well as castrated male and female conspecific intruders, whereas pharmacogenetic inactivation of the region suppresses naturally occurring aggression (Lin et al., 2011). Despite the confluence of these results, the precise role of the hypothalamus in promoting aggression remains debatable. Does it serve as a specialized sensory relay to detect malespecific signals? Is it a premotor area to initiate motor plans related to attack? Or does it play a more complex role, such as setting the general aggressive arousal state?

Some evidence suggests that hypothalamic stimulation drives aggression via a fixed motor plan. For example, hypothalamic stimulation-induced attack usually appears quickly and dissipates within seconds after stimulation is terminated, and certain aggressive movements observed during natural aggression are absent from stimulation-evoked attack in rats (Siegel et al., 1999). Stimulation can also sometimes drive attack toward nonthreatening targets that are not normally the targets of aggression (Siegel et al., 1999; Lin et al., 2011). However, hypothalamic stimulation activates the hypothalamic-pituitary adrenal axis and increases stress hormone release even in the absence of a social target or any overt motor responses (Kruk et al., 2004). Hypothalamic stimulation can also induce animals to actively seek out a suitable target for attack (Roberts and Kiess, 1964), suggesting a more complex role. 
Despite the wealth of functional manipulation data generated in the hypothalamus, few studies attempted to understand its function through electrophysiology. We recently performed chronic in vivo recordings of VMHvl activity in male mice, demonstrating differential responses to males and females: although agonistic interactions with males induce increased VMHvl activity in male mice, sexual interactions with females cause suppression of VMHvl activity (Lin et al., 2011). Here we further analyze these and other data to quantify the relationship between VMHvl activity and sensory and movement parameters during male interaction. Our analysis reveals that VMHvl neurons not only respond strongly to ongoing male-derived olfactory inputs and attack related movements but also predict several parameters of future attacks. Thus, we suggest that the VMHvl plays a role in transforming relevant sensory cues into motivational signals that predict upcoming aggressive events. This finding represents a step toward understanding hypothalamic functioning during complex social interactions.

\section{Materials and Methods}

\section{Animals}

All test animals used in this study were adult proven breeder C57BL/6 male mice (Charles River Laboratories). Mice were singly housed under a reversed $12 \mathrm{~h}$ light/dark cycle for at least 1 week before the test. Stimulus animals were group-housed adult BALB/c or C57BL/6 male, female, and castrated male mice (Charles River Laboratories or The Jackson Laboratory). The care and experimental manipulation of the animals was performed in accordance with National Institutes of Health guidelines and approved by the California Institute of Technology Institutional Animal Care and Use Committee.

\section{Electrophysiological recording and behavior annotation}

Methods for physiological recording in freely moving animals were described previously (Lin et al., 2011). Briefly, a movable bundle of 16 tungsten microwires ( $13 \mu \mathrm{m}$ in diameter each; California Fine Wire) was implanted into the VMHvl during stereotactic surgery. Two weeks after initial implantation, on days of recording sessions, a light-weight head stage was attached to the implanted electrode, and signals were streamed into a commercial acquisition system through a torqueless, feedbackcontrolled commutator (Tucker Davis Technology). During recordings, test animals remained in the home cage and were allowed to freely interact with sequentially presented intact and anesthetized males, females, castrated males, novel objects (e.g., a clean dish or a plastic tube), mouse urine presented on a sterile Petri dish, and food (peanuts or crackers). Anesthetized males were immobilized with an injection of $0.3 \mathrm{cc}$ of a ketamine $(100 \mathrm{mg} / \mathrm{kg})$ and xylazine $(10 \mathrm{mg} / \mathrm{kg})$ mixture. Stimulus presentation order was pseudorandomized, although similar stimuli (e.g., intact male and anesthetized male) were not presented sequentially. Each recorded stimulus presentation consisted of a brief period alone in the home cage ( pre-interaction phase, $1-5 \mathrm{~min}$ ), followed by interaction in the home cage (during-interaction phase, 4-15 min), followed by stimulus removal (post-interaction phase, $1-5 \mathrm{~min}$ ). Stimuli for each type (e.g., a male mouse) were presented multiple times across the recording session, and responses across stimulus class were pooled for analysis. Digital infrared video recordings of animal behavior from both side- and top-view cameras were simultaneously recorded at $640 \times 480$ pixel resolution at 25 frames/s (Streampix; Norpix). Video frame acquisition was triggered by a transistor-transistor logic pulse from the acquisition system to achieve synchronization between the video and the electrophysiological recording. Spikes were sorted using commercial software (OpenSorter; Tucker Davis Technology) based on principal component analysis. Unit isolation was verified using autocorrelation histograms. To consider the recorded cell as a single unit, we imposed four criteria: (1) cells had to have a signal-to-noise ratio $>3$; (2) spike shape had to be stable throughout the recording; (3) the response had to be repeatable during multiple stimulus presentations; and (4) the percentage of spikes occurring with interspike intervals $<3 \mathrm{~ms}$ (the typical refractory period for a neuron) in a continuous recording sequence had to be $<0.1 \%$. Each brain location was recorded one to three times before the screw was carefully adjusted to advance the electrode by $70 \mu \mathrm{m}$. This was necessary because of the extremely small size of the mouse VMHvl subnucleus. Our analysis included recordings from 18 unique locations in five mice obtained on 36 recording days. If the same location was recorded on 2 separate days, it was only included for analysis if a minimum of $3 \mathrm{~d}$ had passed between recordings, to minimize the chance that individual neurons would be recorded more than once; in general, patterns of cell activities across channels were different between these adjacent sessions.

Of the 301 initially recorded units $(n=18,81,12,71,119$ neurons for animals 1 through 5, respectively), 232 were identified as well isolated single units. The 69 remaining neurons were classified as multiunits. Although absolute firing rates for multiunits were slightly but consistently higher in magnitude than their single-unit counterparts, we found no qualitative difference between the response profiles of single units and multiunits during 15 relevant behaviors tested $(p>0.05$, paired $t$ test for each behavior tested independently), and thus units were pooled for analyses. Additionally, we repeated each analysis using single units and found no qualitative difference in results compared with those performed based on combined single and multiunits (data not shown).

Custom software written in MATLAB (Mathworks) was used to facilitate manual annotation of mouse behavior using side- and top-view videos (http://vision.ucsd.edu/ pdollar/toolbox/doc/index.html). A total of 10,000 min of videos was carefully analyzed on a frame-by-frame basis to score relevant behaviors. Custom tracking software written in MATLAB was used to determine the instantaneous positions of the recorded mouse and other social stimuli based on top-view videos (Dollar et al., 2010). These locations were also used to compute instantaneous measures of inter-animal distance, velocity, and acceleration. For social interactions, investigation was defined as nose-to-face, nose-to-body, or nose-to-urogenital contact. For object and urine stimulus presentations, investigation was defined by nose contact to the foreign body. Attacks were defined by a suite of actions initiated by the resident toward the male intruder, which included lunges, bites, tumbling, and fast locomotion episodes between such behaviors. Episodes of attack and investigation have readily distinguishable movement parameters: although both behaviors occur when the resident is close to the intruder, the animals' velocity is much higher during attack. Attack episodes had a mean distance of 73.0 pixels and a mean velocity of 12.9 pixels/frame, whereas investigative episodes had a mean distance of 99.7 pixels and a mean velocity of 4.1 pixels/frame. Behavior was classified as general "nonsocial" if animals were not displaying relevant social behaviors or other specific nonsocial behaviors, such as eating, drinking, or grooming. Circle, chase, and approach are within the social behavior repertoire but occur only rarely. Thus, they are not analyzed in detail here and are included in the "other" category (Fig. 1A).

\section{Physiology analysis}

General firing rate analysis. All analyses were done using custom-written software in MATLAB. The activity matrix was computed using mean subtracted activity, defined as the mean activity for each behavior minus the average of the mean across all behaviors, and sorted based on the activity during attack. To construct the dendrogram, we performed hierarchical clustering using the mean subtracted activity matrix by calculating the linkage between groups based on the Euclidean distance between mean subtracted activity (using the "linkage" function in MATLAB). Cross-validation of clustering was performed by iteratively halving the data and reclustering and was further validated through use of a secondary $k$-means algorithm.

Peristimulus time histograms (PSTHs) for individual behaviors were computed using $50 \mathrm{~ms}$ bins with Gaussian smoothing, unless otherwise noted. When shown, error bars plotted are mean \pm SEM. Neurons were considered to be acutely attack and investigation selective if the activity during investigation or attack was significantly greater than the activity during interleaved nonsocial periods for that same social stimulus type $[p<0.05, t$ test with false discovery rate (FDR) of 0.05$]$. Male responsive neurons were defined as having significantly increased activity during investigation of males, significantly increased activity during attack of 
A

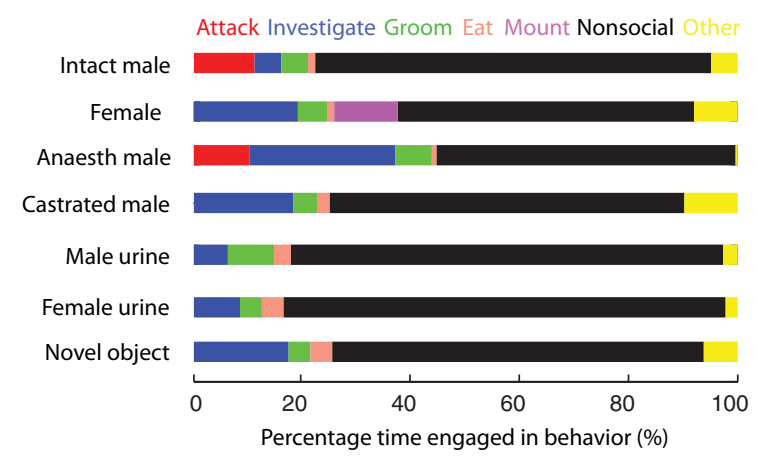

B

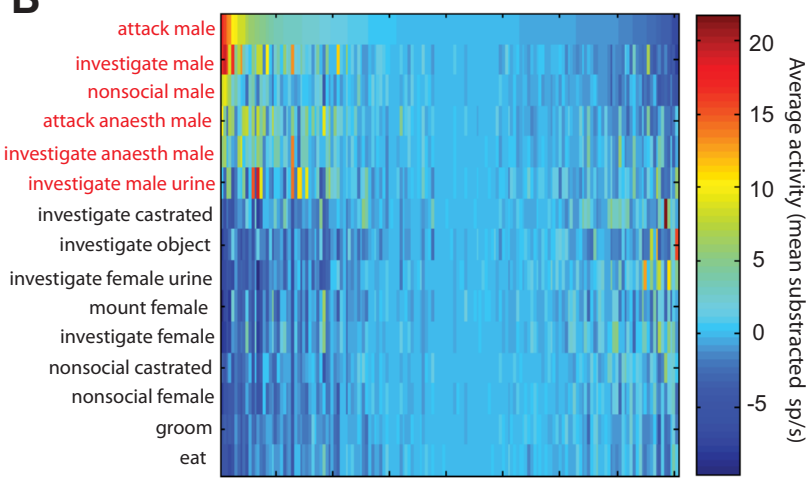

Cell Number

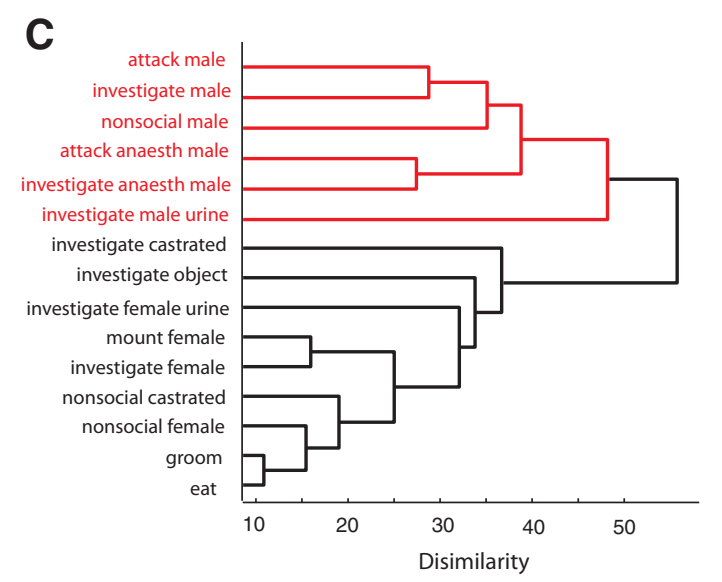

Figure 1. VMHvl response during social and nonsocial interactions. $A$, Schematic of time spent in social and nonsocial behaviors in the presence of each stimulus class (percentage of total time). $\boldsymbol{B}$, Response profiles of VMHvl neurons shown across all stimulus conditions. Heat map shows average mean subtracted firing rate of each VMHvl neuron ( $x$-axis) during each behavior ( $y$-axis). Neurons are sorted left to right in descending order according to their average response during attack male $(n=161$ neurons tested across all stimulus conditions in 5 animals). C, Dendrogram of social and nonsocial behaviors according to hierarchical clustering of activity matrix shown in $\boldsymbol{B}$ exhibits two primary clusters. Male-specific behaviors are shown in red, and non-male behaviors are shown in black.

male intruders, or significantly increased activity during both behaviors relative to activity during nonsocial epochs. Change in activity for specific behavioral conditions was computed by subtracting the average activity across interleaved nonsocial periods from the average activity across an individual behavior (i.e., investigation) for each neuron. Comparisons of population activity of firing rates across any specific condition were done using a Wilcoxon's signed-rank test on averaged raw firing rates or activity change relative to nonsocial epochs and compared with a Bonferroni's corrected $p$ value threshold.
Comparison of pre-interaction and post-interaction epochs. When multiple stimulus presentations for a given type were recorded on the same day, the presentation with the longest post-male period was used to allow for maximal dissipation of signal. We calculated the change in average firing rate of the during-interaction phase and post-interaction phase in reference to the pre-interaction epoch across neurons but within stimulus type. Distributions of spike rate change of during and post periods were assessed for significance using a Student's $t$ test.

Construction of distance-velocity maps. Distance-velocity maps were computed for each neuron based on raw firing rates controlled by distance and velocity. We used the length of an individual video frame as the time bin for this analysis $(40 \mathrm{~ms})$. For each frame, the instantaneous distance between the recorded animal and the stimulus was encoded in pixels. The size of the video frame is $640 \times 480$ pixels; the animal is $\sim 100$ pixels in length excluding the tail, and the cage size is $\sim 500 \times 260$ pixels. The instantaneous velocity of the recorded animal was calculated as the difference in animal position between the last and current frame (pixels per $40 \mathrm{~ms}$ ). Bins had absolute bounds with 50 pixel spacing for distance and 5 pixels/frame spacing for velocity. Population average distancevelocity maps were constructed by averaging maps of individual neurons at each bin. Distance and velocity tuning curves were computed using mean subtracted activity (e.g., mean activity for a neuron across all values of distance) without respect to the other variable (e.g., distance is computed without sorting for velocity). Importantly, the tuning curve is not the average across the distance or velocity map because variable numbers of data points contribute to each bin in the map. Tuning curves constructed from raw activity were not qualitatively different from mean subtracted activity. Significance of distance and velocity tuning was assessed using Kruskal-Wallis ANOVA.

Correlating firing rate to attack duration or interval. Each attack event constitutes a trial. For each neuron, trial-to-trial correlations were computed based on onset response $\left(R_{\text {onset }}\right)$ or response before attack $\left(R_{\text {before }}\right)$ associated with each attack event. The $\mathrm{R}_{\text {onset }}$ was calculated as the average firing rate -500 to $500 \mathrm{~ms}$ around attack onset; $\mathrm{R}_{\text {before }}$ is based on the average firing rate $1000 \mathrm{~ms}$ before the onset of attack. The inter-attack interval was defined as the natural logarithm of the time elapsed from last attack offset to current attack onset. For each neuron, we calculated the Pearson's product moment correlation coefficient $\left(r_{\text {duration }}\right)$ between $\mathrm{R}_{\text {before }}$ and attack duration and the relevant $p$ value across all attack events recorded for the neuron. Similarly, $r_{\text {interval }}$ was calculated between $\mathrm{R}_{\text {onset }}$ and attack interval. At the population level, we determined whether the distribution of the correlation coefficients for male responsive neurons was significantly different from 0 using a $t$ test. For purposes of visualization (see Fig. $6 A, D$ ), attack episodes for each neuron were divided into quartiles within each neuron, based on the inter-attack interval or attack duration and averaged across neurons to produce the PSTH; this separation was not used for calculation of $r_{\text {interval }}$ and $r_{\text {duration }}$.

Attack latency analysis. To examine the relationship between attack latency and instantaneous firing rate, we binned each neuron by time (bin size of $1 \mathrm{~s}$ ) and assigned each bin a corresponding attack latency based on the time from each current bin until the next attack. For each neuron, we calculated the $r_{\text {latency }}$ and $p$ value for vectors of instantaneous firing rates and instantaneous attack latencies for all time bins excluding attack period. To construct the distance-velocity heat map during shortlatency and long-latency periods, we extracted the distance, velocity, activity, and latency to next attack for each video frame. Frames were divided into short-latency (latency $<$ median latency) and long-latency (latency $>$ median latency) trials, and average activity was plotted separately as a function of distance and velocity for both short- and longlatency trials and then averaged across neurons. The difference map (short latency - long latency) was computed for each neuron and then averaged across neurons.

Parameter selection and linear regression analysis. We modeled the activity of each neuron using linear regression and determined parameters using an L1-regularized cost function (Tibshirani, 1996). For each neuron, we computed a vector of spiking activity across the entirety of the inter-male encounter in bins of $1000 \mathrm{~ms}$ and created a series of potential regressor vectors from relevant behavioral variables associated with these time bins. Movement parameters consisted of inter-male distance, resi- 
dent and intruder velocity, and resident and intruder acceleration. Aggression timing parameters consisted of latency to attack, latency from the previous attack, and elapsed time from introduction of the intruder. Aggression-irrelevant parameters consisted of latency to next groom and latency from the previous groom. To determine which of these variables has the strongest effects in the model, we used the lasso algorithm (Tibshirani, 1996). The lasso removes redundant predictors by constraining the L1 norm of the optimal parameter values to be less than an arbitrary penalty. This penalty is zero for the maximally "sparse" model, which contains no regressors, and infinite for the "full" model which corresponds to linear regression. We chose the penalty by determining the sparsest model with the best predictive power (within $1 \mathrm{SE}$ of the minimum mean squared error) on cross-validated data (fivefold crossvalidation). This procedure was applied to each neuron to determine its nonzero parameters and associated regressors. We examined whether parameters were likely to contribute to neural activity in a consistent direction (i.e., positively or negatively correlated) by comparing the distribution of nonzero parameters selected by the lasso regression with the null distribution ( $t$ test).

Using the five parameters most commonly included in the sparse lasso model, we assessed the significance of coefficients using a generalized linear model with the following form:

Firing rate $=(A \times$ Distance $)+(B \times$ Resident Velocity $)+$

$$
(C \times \text { Intruder Velocity })+(D \times \text { Attack Latency })+
$$

$$
(F \times \text { Elapsed Time })+\text { constant. }
$$

We fit coefficients $A-F$ for each neuron and assessed the significance of each coefficient using an $F$ test, which determines whether an individual regressor has an effect after accounting for the effects of all other regressors in the model.

\section{Results}

We recorded and analyzed the activity of 301 neurons in the VMHvl from five C57BL/6 adult male mice in their home cages. Among these neurons, 104 cells were included in a previous report (Lin et al., 2011) but not analyzed in the detail reported here. Recording sites were confirmed histologically after completion of electrophysiological data collection (Lin et al., 2011). Only animals (5 of 30) with all electrodes confined within the VMHvl were used for analysis. During each recording session, the recorded mouse was tested with a wide variety of social and nonsocial stimuli in its home cage. Social stimuli used in the study were male, female, anesthetized male, or castrated male mice. Nonsocial sensory stimuli included food, novel objects, and male or female mouse urine. Simultaneously acquired side- and top-view videos were manually annotated on a frame-by-frame basis to detect specific behaviors, including investigation, attacking, mounting, grooming, and eating. Additionally, the instantaneous positions of the resident and intruder mice were tracked offline using top-view video with custom software, which was then used to determine inter-animal distance, as well as the instantaneous velocity and acceleration of resident and intruder mice. Differences in the number of units included in each analysis reflect exclusion of some neurons as a result of insufficient numbers of trials for particular behaviors (e.g., attack or groom). Several sessions did not yield reliable tracking information, so neurons recorded during these sessions were eliminated whenever tracking data was required for analysis.

To initially characterize the frequency of behaviors, we examined the distributions of specific behaviors during social and nonsocial interactions across animals (Fig. $1 A$ ). In the presence of social stimuli, resident mice spend $10-20 \%$ of the interaction phase attacking, mounting, and investigating these social stimuli (Fig. 1A, red, magenta, and blue, respectively). When not en- gaged in social interaction, animals spent the majority of time engaged in general nonsocial behaviors (Fig. 1A, black) or performing specific nonsocial actions, such as grooming or eating (Fig. 1A, green, orange). Behaviors designated as other include those that were difficult to classify or were rare, such as chasing and circling.

The animals tested were all highly aggressive during interactions with intact males, with the average rate of attack across animals ranging from 2.52 to 7.90 attacks/min. The average number of attacks (reported as mean \pm SEM) across recording sessions was $14.20 \pm 2.78$ attacks (animal $1, n=15$ sessions), $23.38 \pm 3.72$ attacks (animal 2, $n=16$ sessions), $54.57 \pm 4.37$ attacks (animal 3, $n=3$ sessions), $32.79 \pm 5.19$ attacks (animal 4, $n=14$ sessions), and $29.86 \pm 5.68$ attacks (animal 5, $n=14$ sessions). Because of these high levels of aggression across animals, the goal of these experiments was not to quantify individual differences in aggression but instead to examine how neural activity is related to the generation of instantaneous behavior across multiple stimulus conditions.

\section{VMHvl neurons are maximally responsive during male-male social behaviors}

We analyzed the average firing rates during each of 15 social and nonsocial behaviors of recorded neurons in the VMHvl (Fig. 1A). Relevant social behaviors included attack and investigation of a male intruder, attack and investigation of an anesthetized male, investigation of a castrated male, and mounting and investigation of a female. Nonsocial behaviors included non-interactive epochs in the presence of a male, castrated male, or female, as well as investigation of a source of either male or female mouse urine or a novel object introduced into the home cage. The average firing rate of $\mathrm{VMHvl}$ neurons across all behaviors was low (mean \pm SEM firing rate, $3.23 \pm 0.27$ spikes/s; $n=301$ ), and the average activity in the home cage during the prestimulus epoch was even lower (mean \pm SEM firing rate, $2.77 \pm 0.27$ spikes $/ \mathrm{s} ; n=301$ ), but individual neurons exhibited increased activity during specific behaviors. For neurons that were tested across all 15 analyzed behaviors $(n=161)$ during both social and nonsocial conditions, we plotted the average activity of each behavior across neurons after subtracting the mean activity across all behaviors within neurons (Fig. $1 B$ ) and used a binary hierarchical clustering algorithm to explore the relationship between firing rate change and individual behaviors (Fig. 1C). The height of the split between clusters along the $x$-axis represents the dissimilarity between the behaviors connected. The behaviors could be separated into two key clusters based on the activity of the neurons (Fig. 1C). One cluster contained all of the behaviors during male interactions, including aggressive and investigative interactions with both intact and anesthetized males, nonsocial epochs while males were present, and interactions with male mouse urine. The other key cluster contained all remaining behaviors, including interactions with females and castrated males, as well as nonsocial behaviors, such as interacting with an object or female mouse urine, eating, and grooming. Cluster stability was assessed through cross-validation. Neural data were iteratively halved and reclustered using only half of the data. Of 50 clustering trials, 46 of 50 exhibited an identical split between male and non-male associated stimuli. Deviations in 4 of 50 trials were modest and consisted only of changes in clustering for nonsocial male (three trials) or investigate male urine (two trials), which grouped with the non-male cluster. In addition, identical 
A
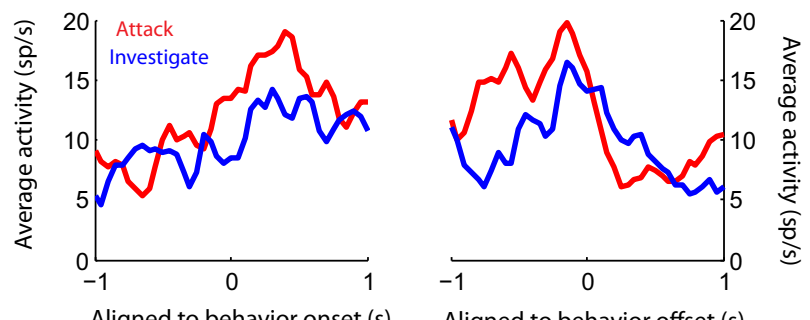

Aligned to behavior onset (s)

Aligned to behavior offset (s)
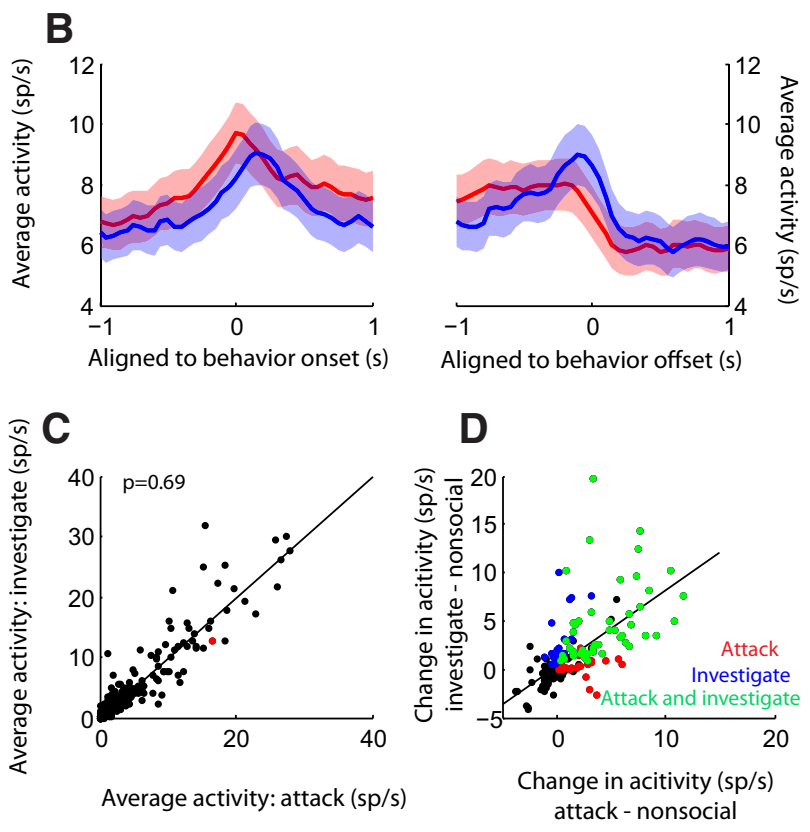

Figure 2. VMHvl neurons transiently increase activity during attack and investigation of male mice. $\boldsymbol{A}, \boldsymbol{B}$, PSTH of an example neuron $(\boldsymbol{A})$ and population average $(\boldsymbol{B})$ of activity of male responsive neurons during attack (red) and investigation (blue) shown aligned to the onset (left) and offset (right) of behavior. ( $n=105$ neurons in 5 animals). Error bar is SEM, and bin width for all PSTHs is 50 ms with Gaussian smoothing. C, Average VMHvl cell activity during attack male is not significantly different from that during investigating male. Red circle indicates example cell shown in $\boldsymbol{A}$. $\boldsymbol{D}$, Distribution of the difference in activity between attack/ investigation and interleaved nonsocial periods of male responsive neurons. Red, blue, and green dots show attack, investigation, and dual responsive cells, respectively $(n=105)$.

grouping was observed using an alternative clustering algorithm ( $k$-means clustering using partitioning into two groups). This behavioral grouping demonstrates that, although neuronal responses across the behaviors tested showed heterogeneity, the pattern of VMHvl activity during encounters with male-related stimuli is readily distinguishable from activity observed during non-male-specific behaviors.

VMHvl cells exhibited acute increases in activity during specific male social behaviors relative to the ongoing activity before the onset of these behaviors. Both in single neurons (Fig. 2A) and across the population (Fig. 2B), we observed that neural activity in a PSTH aligned to specific behaviors increased at the onset of both investigation (Fig. $2 A, B$, blue trace) and attack (Fig. $2 A, B$, red trace) and returned to baseline after the cessation of the behaviors. For each neuron, we compared the spike rate distribution during investigation or attack with the distribution of spike rates of interleaved nonsocial episodes using a $t$ test. One hundred five of 267 (39.3\%) neurons recorded during intact male stimulus presentations exhibited significantly increased activity during attack, inves- tigation, or both ( $t$ test corrected for Benjamini-Hochberg FDR at 0.05). Among these significant male responsive neurons, approximately half showed increases for both behaviors (49.5\%, 52 of 105; Fig. 2D, green dots). A subset (27 of 105 neurons, $25.7 \%$; Fig. $2 D$, red dots) responded only during attack, and 26 of 105 (24.8\%; Fig. 2D, blue dots) increased only during investigation. The mean \pm SEM increase during attack in male responsive neurons was $2.97 \pm 0.27$ spikes/s $(n=79)$, and the mean \pm SEM increase during investigation was $3.54 \pm 0.39$ spikes/s $(n=78)$. Across the population of male responsive neurons, the average activity of VMHvl cells during investigation of male intruders and attack of male intruders was not significantly different (Fig. $2 C ; p=0.693$, Wilcoxon's signed-rank test, $n=105$ ).

Approximately $11.6 \%$ of VMHvl cells showed significantly decreased activity during either male attack or investigation $(t$ test with FDR of 0.05). Responses during male investigation and attack are generally of the same direction; only 8 of 267 cells recorded during interactions with intact males showed opposing response patterns during these two behaviors. The change in activity during attack and investigative behaviors (average activity during investigation or attack minus average activity during nonsocial period) is correlated across the population (Fig. $2 D ; r^{2}=$ $0.423, p \ll 0.001$, least-squares regression). Together, these data suggest that VMHvl activity encodes acute changes in malerelevant sensory information and are not exclusively active during attack.

We found male responsive neurons in each of the five animals we recorded during interactions with intact males (12 of 18, 23 of 79,4 of 10,34 of 59, and 32 of 101 male responsive neurons in animals $1-5$, respectively). Although the extremely small size of the VMHvl makes it difficult to assess anatomical clustering of recorded neurons along the mediolateral axis, we did observe a tendency for a higher proportion of male responsive neurons when implantations were biased toward the posterior part of VMHvl. Two animals with implantations ranging from bregma -1.64 to $-1.88 \mathrm{~mm}$ had a higher percentage of male responsive neurons ( 12 of $18,66.7 \%$; and 34 of $59,57.6 \%$ ), whereas the two animals with the most anteriorly located implantations ranging from bregma -1.34 to $-1.47 \mathrm{~mm}$ had a smaller proportion of male responsive neurons ( 23 of $79,29.1 \%$; and 4 of $10,40.0 \%$ ). This anatomical bias is consistent with the observation that inhibition of the posterior VMHvl has a stronger effect on suppressing inter-male aggression than suppressing the anterior $\mathrm{VMHvl}$ (Lin et al., 2011).

\section{Male responsive neurons in the VMHvl respond to male conspecific olfactory cues}

What drives neurons in the VMHvl to respond when the mouse is investigating a male conspecific? Potential causes include sensory variables, such as the sight and sound of a moving animal, conspecific olfactory cues, and the motor act of investigation. To distinguish which cue(s) drives VMHvl neural responses, we used a series of social and nonsocial stimuli to control for the contribution of sensory inputs of different modalities (Fig. 3A). To control for sensory stimulation of auditory and movement cues, we compared stimulus presentations using anesthetized males versus "intact" moving males, because anesthetized males produce no movement or sound as confirmed by our sound recording of an anesthetized male across a range of frequencies, with and without the presence of the resident male. Castrated males, whose lack of testosterone causes them to have reduced production of male-specific pheromones (Bruce, 1965; Novotny et al., 
A
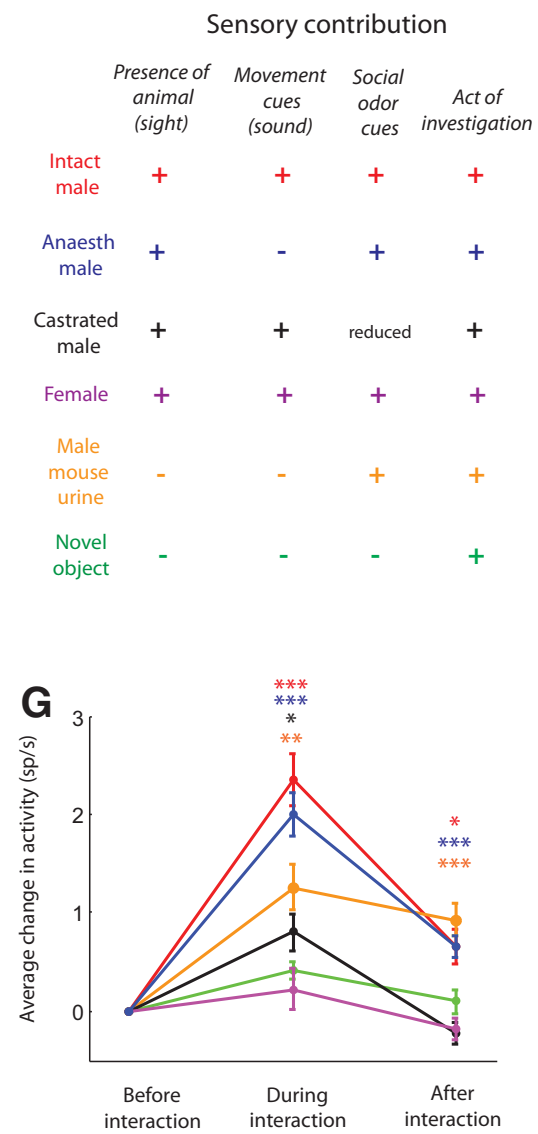

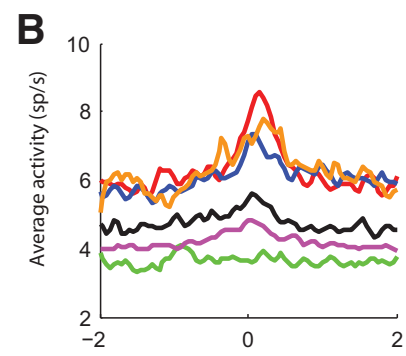

Aligned to investigate onset (s)

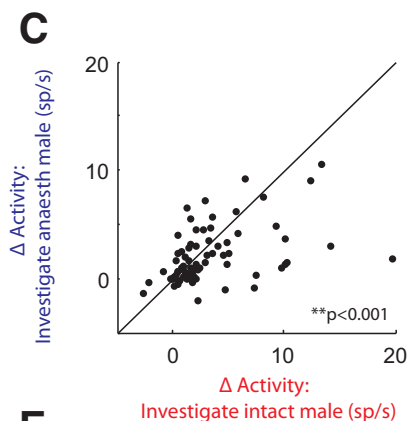

E

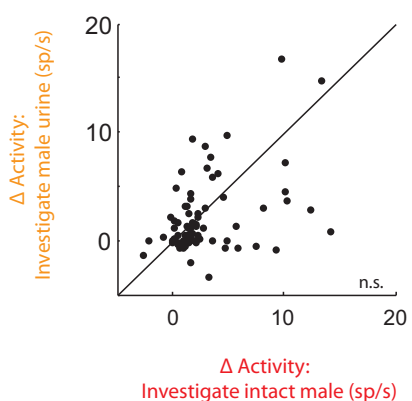

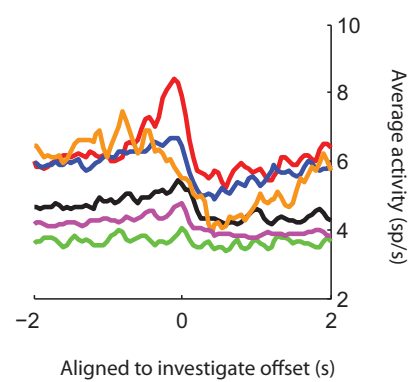

D

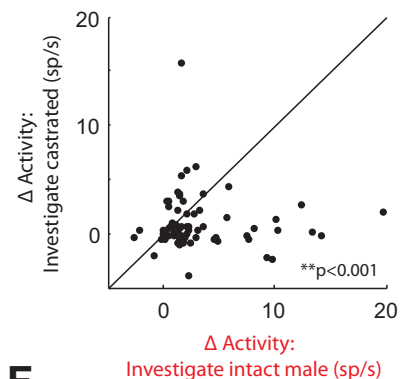

$\mathbf{F}$

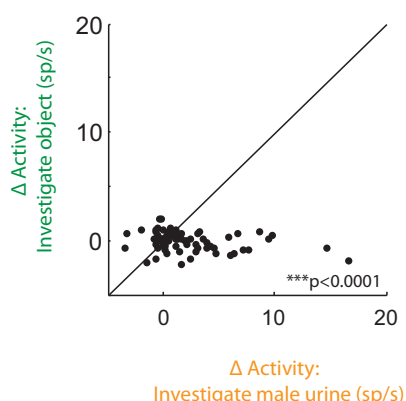

Figure 3. VMHvl neurons respond to male-derived olfactory cues. $A$, Test stimuli to control for sensory modality included intact male (red), anesthetized male (blue), castrated male (black), female (purple), male mouse urine (orange), and object (green). Color conventions are consistent across the panels. B. Population PSTH showing activity of male responsive neurons during investigation of various stimuli listed in $\boldsymbol{A}$. Responses are aligned to investigation onset (left) and investigation offset (right). Bin size is $50 \mathrm{~ms}$. $\boldsymbol{C}-\boldsymbol{F}$, Comparison of activity change in male responsive neurons during investigation of various tested stimuli. Activity change during investigation of an intact male is significantly greater than activity change during investigation of either an anesthetized male $(\boldsymbol{C}, n=82)$ or a castrated male $(\boldsymbol{D}, n=72)$ but is not significantly different from activity change during investigation of a source of male mouse urine $(\boldsymbol{E}, n=75)$. Activity change during investigation of male urine is significantly greater than that during novel object investigation $(\boldsymbol{F}, n=75)$. $\mathbf{G}$, Comparison of averaged activity during pre-interaction, during-interaction, and post-interaction epochs. Activity of male responsive neurons increases during investigation relative to pre-interaction epoch and remains elevated after removal of male-related stimuli but not other stimuli ${ }^{*} p<0.05,{ }^{* *} p<0.01,{ }^{* * *} p<0.001$, one-sample $t$ test using a subpopulation of male responsive neurons tested in each condition; intact male, $n=105$; anesthetized male, $n=79$; castrated male, $n=65$; female, $n=102$; male urine, $n=75$; novel object, $n=92$; all male responsive neurons from $n=5$ animals).

1985), are rarely the subjects of attack, yet they can control for the movement of a social stimulus in the home cage. Interactions with female mice also control for the movement and sound of a social stimulus, but female mice carry distinct odor and social relevance. We also explored neural response to a pure male conspecific odor source (urine in a dish) in the absence of an animal. An object (dish or other novel item) without any odor source was also introduced as a stimulus to control for olfactory unrelated disturbance.

Across the population of male responsive neurons, we observed robust increases in acute activity when resident mice investigated intact males, anesthetized males, and a source of male mouse urine. In contrast, only weak increases in activity were observed during investigation of castrated males or females, and no increase was observed during investigation of a novel object (Fig. $3 B$ ). Across the population, we compared changes in activity relative to nonsocial epochs rather than raw activity because stimulus conditions may result in changes in the nonsocial epochs. In a direct neuron-by-neuron comparison of the change in activity (Fig. $3 C-F$, activity during investigation minus activity during interleaved nonsocial periods) under various stimulus conditions, we found that neurons exhibited a significantly higher change in activity when investigating an intact male compared with investigating an anesthetized male (Fig. $3 C ; p<0.01$, Wilcoxon's signed-rank test, $n=82$ male responsive neurons tested for both intact male and anesthetized male; value is significant using Bonferroni's significance threshold corrected for multiple comparisons, $p<0.0125$ ) or a castrated male (Fig. 3D; $p<0.001$, Wilcoxon's signed-rank test, $n=72$ male responsive neurons tested for intact male and castrated male, $p<0.0125)$. In contrast, we found that the average change in activity was not significantly different between investigation of an intact male and male mouse urine (Fig. $3 E ; p>0.05, n=75$ for male responsive neurons tested for both intact males and male mouse urine), although individual neurons may respond selectively. Increased activity during investigation of male urine cannot be attributed to cage disturbance or the motor action of investigation, because the change in activity was significantly higher when mice investigated the urine source compared with a novel object (Fig. $3 F ; p \ll$ $0.001, n=75$ for male responsive neurons tested for both male 
A

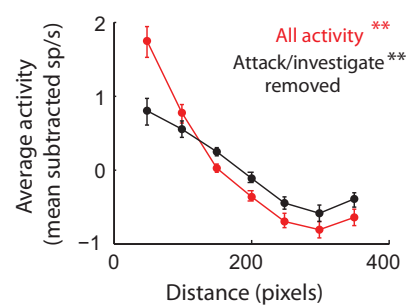

E

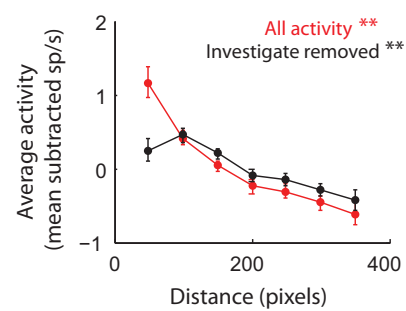

B

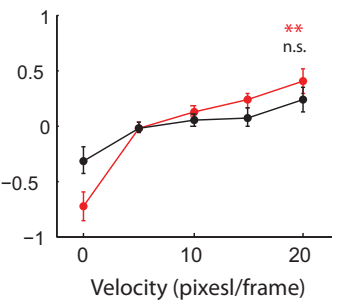

F

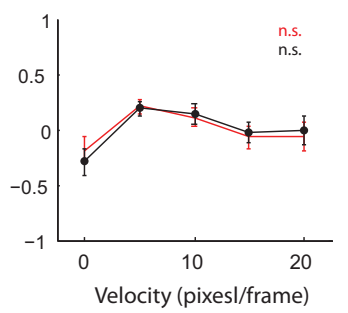

C

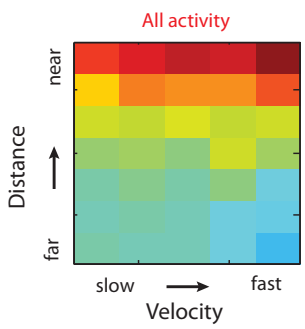

Attack/investigate removed

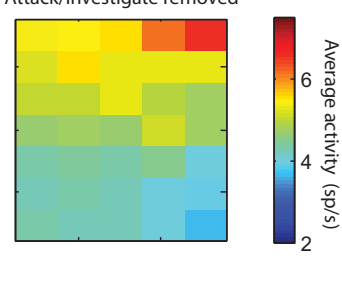

H

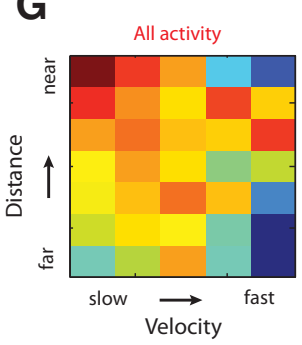

Investigate removed

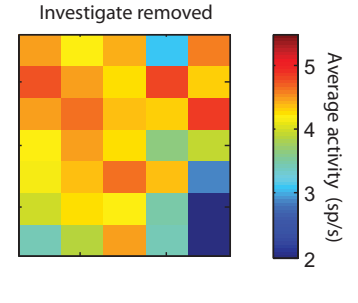

Figure 4. VMHvl activity of male responsive neurons is modulated by inter-male distance and movement velocity during attack. $A, B$, Mean subtracted firing rate as a function of distance $(A)$ and velocity $(\boldsymbol{B})$ for all periods (red) and periods excluding investigation and attack (black). Cell activity is significantly tuned to both parameters when all periods are included, although only distance remains tuned after removing attack and investigation epochs. $\boldsymbol{C}, \boldsymbol{D}$, Average firing rates of male responsive cells plotted as a function of the distance ( $50-400$ pixels using 50 pixel spacing) between the resident and the male intruder and the instantaneous velocity ( 0 - 30 pixels/frame using 5 pixels/frame spacing) of the resident mouse for all time bins during inter-male encounters $(\boldsymbol{C})$ or all time bins excluding investigating and attacking male (D). $\boldsymbol{E}-\boldsymbol{H}$, Male responsive neurons are also tuned to the distance from a source of male mouse urine even when investigation episodes are excluded, but activity is not modulated by movement velocity during male urine trials. Male responsive neurons are from all five animals ( ${ }^{* *} p \ll 0.01, n=95$ neurons in $\boldsymbol{A}-\boldsymbol{D}, n=75$ neurons in $\boldsymbol{E}-\boldsymbol{H}$ ).

mouse urine and object, $p<0.0125$, Bonferroni's corrected threshold).

The increased response of VMHvl to an intact male compared with a castrated male suggests that male-specific olfactory cues play an important role in driving VMHvl activity. Our observation that male responsive neurons can also respond to male mouse urine further confirms this conclusion. However, we observed small but significantly higher activity during intact male investigation than anesthetized male investigation, suggesting that conspecific sensory cues operating through other modalities (such as hearing and vision) may also play a minor role in modulating VMHvl activity.

Do changes in activity to male-specific stimuli persist after the removal of the stimulus? To address this, we compared the activity in the post-interaction phase, interaction phase, and preinteraction phase. We found that, not only was activity during interaction increased for male stimuli, VMHvl activity was consistently higher during the post-interaction epoch relative to the pre-interaction epoch after removing male-related stimuli [Fig. $3 G$; paired $t$ test to compare the before and during and before and after interaction periods, for intact male: red, $t_{(\mathrm{df})}=104, p \ll$ 0.001 during, $p<0.05$ after; for anesthetized male: blue, $t_{(\mathrm{df})}=$ $78, p \ll 0.001$ during, $p<0.001$ after; orange: $t_{(\mathrm{df})}=74, p<$ 0.005 during, $p \ll 0.001$ after]. Although introducing a castrated male resulted in a small but significant increase during interaction (Fig. $3 G$, during interaction, black; $t_{(\mathrm{df})}=64, p<0.01$ ), this activity did not remain elevated after intruder removal (postinteraction, $p=0.220$ ); rather, there was a trend toward reduced activity in the post-interaction phase. Finally, introducing or removing a female or a novel object into the animal's cage caused no change in firing after stimulus removal in male responsive neurons [Fig. $3 G$, magenta for female $\left(t_{(\mathrm{df})}=101, p=0.317\right)$, green for object $\left.\left(t_{(\mathrm{df})}=91, p=0.613\right)\right]$.

\section{VMHvl activity is tuned to inter-male distance and attack-related movement}

Because neurons in the VMHvl respond robustly to an odor cue in the absence of a social stimulus, one possibility is that changes in neural activity during attack reflect fluctuating sensory input from male conspecifics. Alternatively, the responses of $\mathrm{VMHvl}$ neurons may also be sensitive to aggression-specific movements during inter-male interactions. To examine the relationship between sensory input, motor output, and VMHvl activity in more detail, we extracted the instantaneous inter-male distance and the instantaneous movement velocity for each recording session during male-male interactions. Although velocity fluctuates as the animal moves around the cage, attack motion such as lunging and tumbling consistently corresponds to moments of increased velocity. If olfactory input were the sole determinant of firing rate change during attack, VMHvl activity should covary only with distance (not velocity). Alternatively, if VMHvl firing rate correlates with both distance and velocity, factors other than changes in sensory input contribute to VMHvl activity during attack. We plotted the average spiking activity change as a function of distance (Fig. $4 A$ ) or velocity (Fig. $4 B$ ) and found that the firing rate is significantly correlated with both parameters, suggesting that response during attack cannot be fully accounted by sensory input alone (Fig. $4 A, B$, red curves; $p \ll 0.001$ for distance and velocity, Kruskal-Wallis ANOVA, $n=95$ male responsive neurons with accurate tracking information). Furthermore, we constructed a heat map to reveal how the instantaneous firing rate averaged across all male responsive neurons varies with both velocity ( $x$-axis) and inter-male distance ( $y$-axis) and found that the averaged activity is maximal when the distance between the mice is the least and when velocity is highest (Fig. 4C), which corresponds to both investigation and attack episodes. To determine whether the same relationship between firing rate and ve- 
locity or distance holds true in the absence of relevant aggressive behaviors that acutely increase VMHvl activity, we replotted the data for the period excluding inter-male investigating and attacking and found that male responsive neurons were still strongly positively tuned to the distance between animals (Fig. $4 A, D$, black curve; $p \ll 0.001$ ) yet were no longer significantly tuned to velocity (Fig. $4 B, D$, black curve; $p=0.70)$. These data demonstrate that increased activity during attack is not only attributable to decreased inter-male distance but also to attack-related movement variables.

Our findings that VMHvl neurons are modulated by the distance from relevant sensory input and not by nonspecific changes in movement velocity were further confirmed by examining activity changes during specific nonsocial stimulus interactions. During interactions with a source of male mouse urine, neuronal activity in male responsive neurons was inversely correlated with distance to the urine source, even when periods of direct investigation of the urine source were eliminated from analyses (Fig. 4E-H; inter-male distance, $p$ $\ll 0.001$ for all activity and for activity except for investigation, Kruskal-Wallis ANOVA, $n=75$ male responsive neurons with tracking information tested with male mouse urine). However, VM$\mathrm{Hvl}$ activity during nonsocial interaction with a source of male mouse urine is not tuned to the velocity of the resident mouse ( $p=0.251$ for all activity, $p=0.138$ for all activity excluding investigative epochs). Although the responses of VMHvl neurons are tuned to distance but not velocity during interactions with male urine, activity of VMHvl neurons is tuned to neither distance nor velocity during interactions with a novel object (for distance, $p=0.333$ and for velocity, $p=$ 0.093, Kruskal-Wallis ANOVA test), demonstrating that proximity to a distinct odor cue but not an object in the cage can modulate VMHvl neural activity.

VMHvl activity is correlated with parameters related to future and past attack events

During inter-male encounters, the initiation of attack is often preceded by an investigative phase. Across our recorded behavioral sessions, $20.4 \%$ of attacks occurred directly after an investigation episode, although in the majority of cases, investigation was followed by a transition into a nonaggressive nonsocial epoch. If neurons in the $\mathrm{VMHvl}$ were merely reporting the specific inter-male distance without respect to any future aggressive episode, then the response during investigation should be similar, regardless of whether the animal attacks in the immediate future. Alternatively, if VMHvl activity signals information about the likelihood of future attack, then the cells may respond more vigorously when investigation is followed by attack. For neurons that significantly increased their response during investigation relative to nonsocial epochs $(n=78$ investigate male compared with interleaved nonsocial, $t$ test with FDR of $<0.05$ ), we sorted neural activity during investigation episodes based on whether each investigative episode was followed by an attack or a nonsocial behavior. We plotted the corresponding PSTHs aligned to the onset or offset of investigation for these two classes of investigative episodes (Fig. 5A) normalized by the average activity across both conditions. Activity in the VMHvl increased at the onset of investigation for both investigation leading to attack and investigation leading to nonsocial behaviors; however, when investigation preceded attack, the onset activity significantly increased relative to investigation leading to nonsocial behavior (Fig. $5 \mathrm{~A}$, left). At the offset of investigation, if investigation transitions into attack, VMHvl activity continues to rise, but if investigation transitions into nonsocial behavior, activity returns to preinvestigation baseline (Fig. $5 \mathrm{~A}$, right). Across the population, average responses during investigative episodes that preceded attack were significantly higher than those during episodes preceding a nonsocial behavior, for both the onset of the investigative episode (Fig. $5 B$, red dots; $250 \mathrm{~ms}$ after onset, $t_{(\mathrm{df})}=74, p<$ 0.05 , paired $t$ test, mean difference of $1.252 \pm 0.61$ spikes) and the time interval directly preceding the behavioral transition (Fig. $5 B$, black dots; $250 \mathrm{~ms}$ before offset, $\left.t_{(\mathrm{df})}=74, p<0.05\right)$.

The resident mouse's behavior also reflected the likelihood of upcoming attack. Across all behavioral sessions, we observed that the median duration of investigative episodes that preceded attack was significantly longer than the duration of investigations 
A

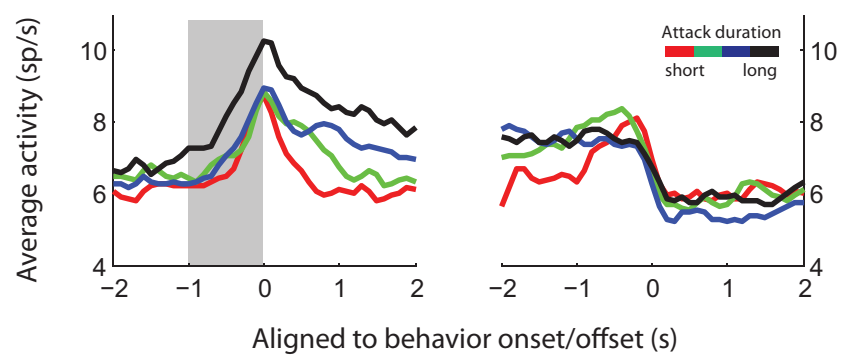

D

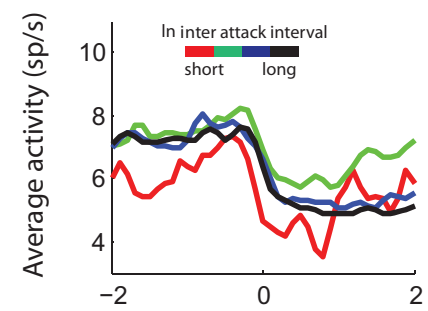

B
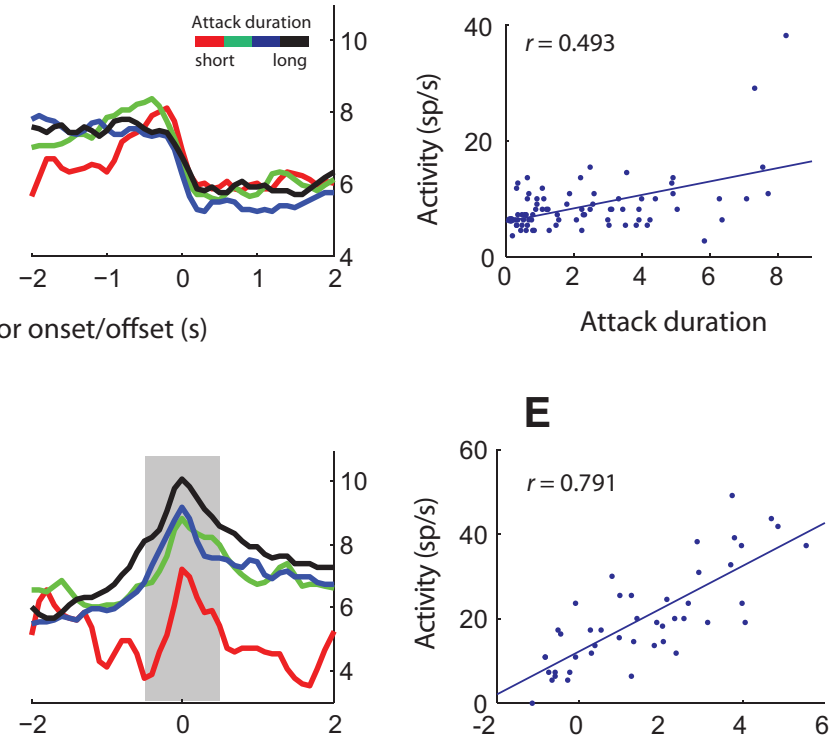

E

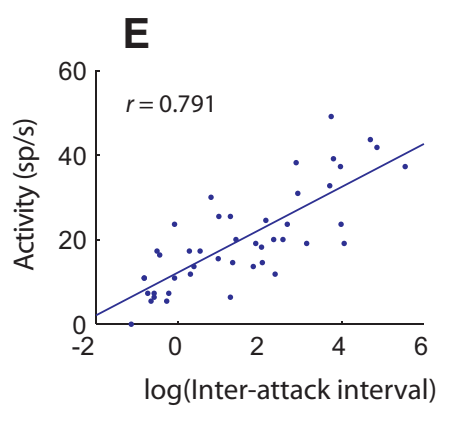

Aligned to behavior offset/onset (s)

C

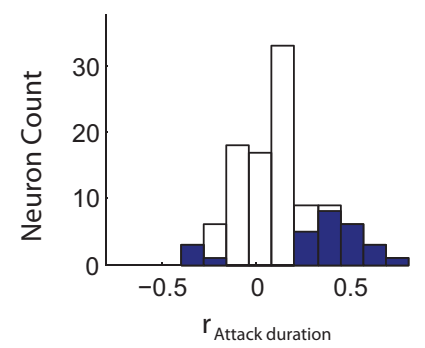

$\mathbf{F}$

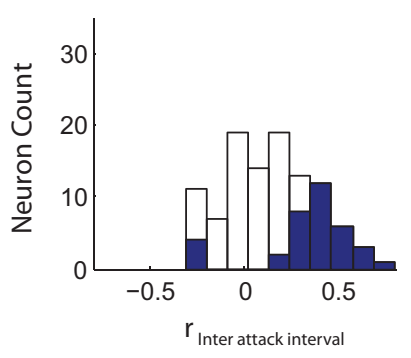

Figure 6. VMHvl activity at attack onset correlates with attack duration and time elapsed from last attack. A, Population average PSTHs of the mean firing rate of male responsive neurons aligned to attack onset (left) and offset (right) for attack trials separated into quartiles within each neuron based on attack duration. Red, green, blue, and black lines indicate PSTHs composed of attack trials lasting the shortest to the longest. Gray bar shows pre-attack activity epoch used in $\boldsymbol{B}$ and $\boldsymbol{C}$. $\boldsymbol{B}$, Trial-to-trial correlation of pre-attack activity (1 s before attack) and attack duration for an example neuron. Line shows the least squares regression. $C$, Histogram of distribution of correlation coefficients $\left(r_{\text {duration }}\right)$ between attack duration and pre-attack response (mean firing rate during -1 to 0 s) for all male responsive neurons. Blue overlay shows cells with significant correlation. $\boldsymbol{D}$, Population average PSTHs of the mean firing rate of male responsive neurons aligned to attack offset (left) and onset (right) for attack trials separated into quartiles based on inter-attack interval. Gray bar shows time window used for analysis in $\boldsymbol{E}$ and $\boldsymbol{F}$. Color conventions as in $\boldsymbol{A}$. $\boldsymbol{E}$, Scatter plot showing trial-to-trial correlation between attack onset activity $(500 \mathrm{~ms}$ before attack to $500 \mathrm{~ms}$ after attack onset) and the In(inter-attack interval) of an example cell. $\boldsymbol{F}$, Histogram showing the distribution of $r_{\text {inter-attack interval }}$ between $\ln$ (inter-attackinterval) and onset response of the next attack. Blue overlay shows cells with significant correlation. For all population analyses, $n=105$ male responsive neurons from 5 animals; PSTH bin width for $\boldsymbol{A}$ and $\boldsymbol{D}$ is $100 \mathrm{~ms}$.

that preceded nonsocial behaviors (Fig. $5 C$; $p<0.001$, Wilcoxon's signed rank, $n=36$ behavioral sessions). This difference in duration does not account for the observed activity difference between investigations preceding attack versus nonsocial behaviors, because we compared only the onset activity for investigations that exceeded a minimum threshold of $250 \mathrm{~ms}$ and analyzed activity from a matched time bin. Instead, it may reflect changes in the internal state of the animal as it prepares to attack.

To further investigate the role of VMHvl in encoding information about future aggressive acts, we examined the relationship between VMHvl activity before attack and attack duration. The duration of an individual attack episode can last from $40 \mathrm{~ms}$ (the length of a video frame) to $>10 \mathrm{~s}$ (mean \pm SEM attack duration, $2.22 \pm 0.07 \mathrm{~s} ; n=1266$ attack episodes) and can vary from a single bite to a prolonged intensive tussle. We plotted the activity of male responsive VMHvl neurons as a function of attack duration (Fig. 6A). We observed that longer attack durations were preceded by larger firing rate increases before the onset of attack and that these increases could be observed as far as $2 \mathrm{~s}$ before attack onset. To quantify this relationship, we extracted the trial-to-trial correlation between the average firing rate across the $1 \mathrm{~s}$ preceding the attack onset and the subsequent attack duration for each neuron in which each attack event constitutes a single trial (mean number of attack events recorded for each neuron is $60.0 \pm 35.49$, ranging from 7 to 174 ; example trial-totrial correlation shown in Fig. 6B). We found that, among 105 male responsive cells, 23 showed a significant correlation $(21.9 \%$, Pearson's correlation with FDR of 0.05); also, the distribution of correlation coefficients $\left(r_{\text {duration }}\right)$ across the population was positively skewed, i.e., dominated by positive correlations (Fig. 6C; $r_{\text {duration }}$ mean of $0.12, t_{(\mathrm{df})}=104, p \ll 0.001$ for the distribution of $\left.r_{\text {duration }}\right)$. The correlation coefficient of significantly correlated neurons, found in all five recorded animals, ranged from 0.273 to 0.801 for positive correlations and -0.394 to -0.221 for negative correlations and were found in all five recording animals. Although neural activity before attack can predict upcoming attack duration, activity declines to a similar level after cessation of attack (Fig. 6A, right). The distribution of correlation coefficients based on attack offset neural activity (average activity $1 \mathrm{~s}$ after attack offset) and attack duration is not significantly different from zero $\left(p=0.787, t_{(\mathrm{df})}=104\right)$.

Male responsive neurons encode information about imminent attacks but not previous attacks; still we hypothesized that activity might be modulated by the time elapsed from a previous attack. When attack episodes were sorted by their inter-attack interval (i.e., time interval from previous attack to current attack), we observed a positive relationship between the time elapsed from the last attack and the onset of VMHvl activity during the current attack, such that longer intervals predicted stronger attack onset responses (Fig. 6D). A significant positive correlation was found between the natural logarithm of interattack interval and VMHvl activity at the onset of the next attack (from -500 to $500 \mathrm{~ms}$ around attack onset; Fig. $6 \mathrm{D}$, gray area) in 21 of 105 male responsive cells $(20 \%$, Pearson's correlation with FDR of 0.05; for an example neuron, see Fig. 6E). Across the population, the distribution of coefficients was significantly positively skewed (Fig. $6 F$; population mean of $0.14, t_{(\mathrm{df})}=104, p \ll$ $0.001)$. The correlation coefficients of significantly correlated neurons ranged from 0.161 to 0.792 for positive correlations and -0.308 to -0.206 for negative correlations and were observed in 
A

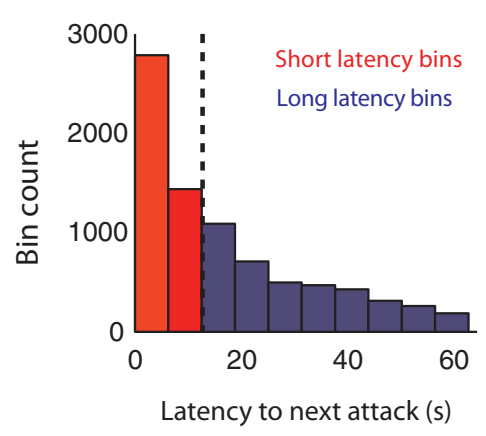

D

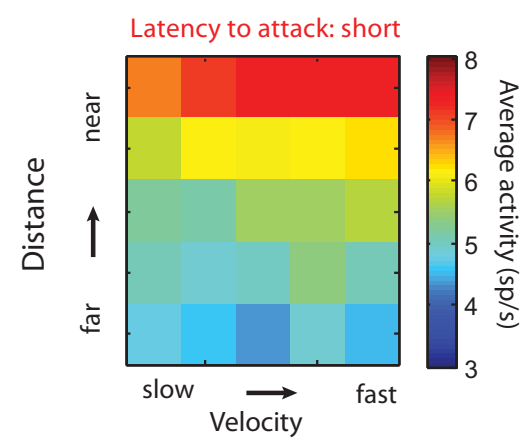

B

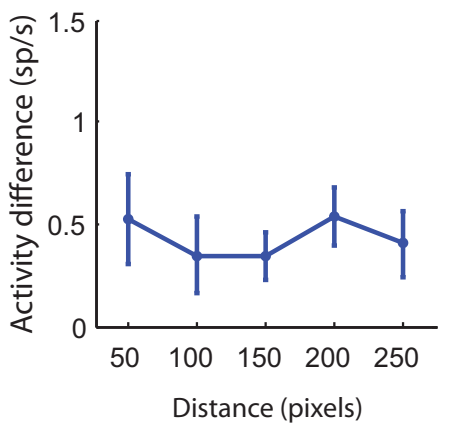

E

Latency to attack: long

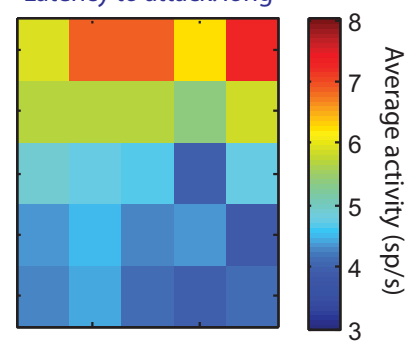

C

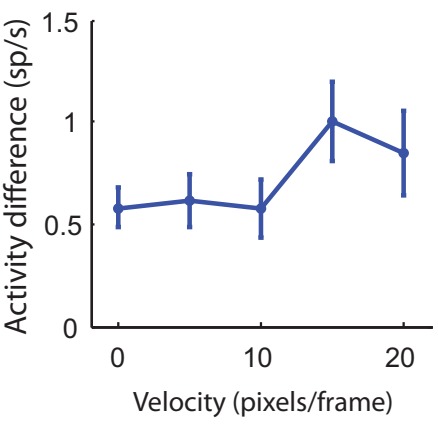

$\mathbf{F}$

Difference: short - long

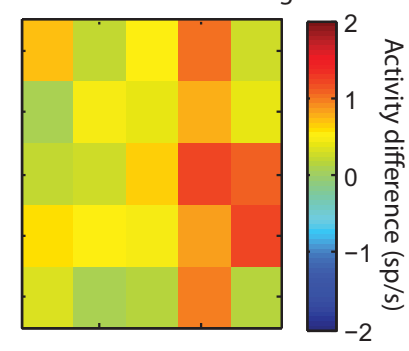

Figure 7. VMHvl activity correlates with latency to next attack independent of changes in inter-male distance and movement velocity. Activity during each inter-male interaction was separated into short- and long-latency attack time bins. $\boldsymbol{A}$, Example separation of time bins (1 s) during single inter-male sessions by median attack latency (dotted line) into short latency to attack (red) and long latency (blue) to attack. $\boldsymbol{B}$ and $\boldsymbol{C}$, Across the population, VMHvl activity increases for short-latency attack time bins relative to long-latency attack bins regardless of inter-male distance ( $\boldsymbol{B}$ ) or movement velocity (C). Error bars show \pm SEM. $\boldsymbol{D}-\boldsymbol{F}$, Heat maps showing the average activity of male responsive cells as a function of distance and velocity during short-latency attack time bins ( $\boldsymbol{D}$ ), long-latency attack time bins $(\boldsymbol{E})$, and the difference between these maps $(\boldsymbol{F})$. Activity differences between short- and long-latency maps were computed for each neuron and then averaged across neurons. ( $n=93$ male responsive neurons in 5 animals for $\boldsymbol{B}-\boldsymbol{F})$.

five of five recorded mice . Furthermore, the duration of the inter-attack interval was also positively correlated with the duration of the subsequent attack (mean $r_{\text {duration-interval }}=0.190, p \ll$ $0.001, t$ test, 11 of 36 recording sessions are individually significant). Together, these data demonstrate that VMHvl activity is strongly correlated with parameters that indicate the timing of past and future attack episodes.

\section{VMHvl activity predicts attack latency independent of velocity and inter-male distance}

In addition to the correlations we observed between VMHvl activity, future attack duration, and the time elapsed from last attack, we also found that the ongoing activity of VMHvl neurons is strongly correlated with the latency to the next attack. We examined the relationship between VMHvl instantaneous activity (200 ms time bin during the whole recording period except for attack and investigate) and the latency to the next attack throughout the testing period. A significant correlation between these two variables was observed in 62 of 105 (59.1\%, Pearson's correlation with FDR of 0.05 ) male responsive neurons, and the majority of these correlations (56 of 62 or $90.3 \%$ of cells) were negative. Significant negative correlations were found in each of the five recorded animals ( 8 of 12,9 of 23,3 of 4,20 of 34 , and 16 of 32 male responsive neurons for animals $1-5$, respectively). Thus, short latencies to attack are correlated with high levels of activity in VMHvl neurons. However, because we also observed that VM$\mathrm{Hvl}$ neurons are highly tuned to the proximity to male sensory cues and also to changes in attack motion, it is likely that some correlation between activity and attack latency can be explained by these variables, because attack often follows a period of close interaction and fast movement toward or around the intruder.

To dissociate whether VMHvl predicts attack latency independent of changes in inter-male distance and velocity, we compared VMHvl activity between time epochs with short and long attack latencies using a temporal window in which inter-male distance and velocity were comparable. Specifically, for each of the 93 male responsive neurons with tracking information, we assigned each time bin during inter-male interaction as either short attack latency (below median latency) or long attack latency (above median) (example distribution of attack latencies for a single session are shown in Fig. 7A). We then calculated the difference in spiking activity between short-latency and longlatency epochs for each neuron as a function of distance (Fig. $7 B$ ) and velocity (Fig. $7 C$ ), averaged across the population. Activity during short-latency attack epochs was consistently higher than activity during long-latency attack epochs, regardless of distance or velocity, suggesting that the negative correlation observed between activity and latency to the next attack was to some extent independent of distance and velocity.

However, these results do not rule out the possibility that increased VMHvl activity during a short-latency epoch at a given inter-male distance is caused by increased velocity during that epoch or vice versa. To investigate this possibility, we examined VMHvl activity as a function of both distance and velocity simultaneously by constructing a 2D activity map for each neuron with velocity as the $x$-axis and distance as the $y$-axis. We extracted a "difference" map (Fig. $7 F$ ) by subtracting the long-latency map (Fig. 7E) from the short-latency map (Fig. 7D) for each neuron 
and averaged across neurons. Importantly, the boundaries of bins in the long- and short-latency maps were identical, and within each distance-velocity bin, no significant difference in distribution was observed for either distance or velocity $(p>0.05$, Kolmogorov-Smirnov test for each bin) such that differences in activity cannot be attributed to within-bin distance or velocity distribution difference. In other words, although distance-velocity maps for short- and long-latency attack epochs both showed increased activity at short distances and high velocities (Fig. $7 D, E)$, subtraction of these maps revealed a distance and velocity-independent positive difference in activity.

\section{Linear regression model}

To demonstrate comprehensively and quantitatively the correlation between VMHvl activity and aggression-specific behavioral parameters, we modeled the spike rate distribution across the inter-male encounter for each neuron using eight aggression-relevant parameters (inter-male distance, resident velocity and acceleration, intruder velocity and acceleration, latency to attack, latency from previous attack, and time elapsed from the introduction of the intruder). Additionally, we included two parameters that may modulate neural activity but are not clearly linked to aggression: latency to groom and latency from previous groom. Although interactions between these variables are likely complex and may modulate activity nonlinearly for individual neurons, our goal was to adequately capture the direction of the effects of variables on overall VM$\mathrm{Hvl}$ activity rather than elucidate the precise contribution of each variable. To this end, we modeled neural activity using generalized linear regression with a lasso regularization method (Tibshirani, 1996). This method imposes a penalty for each additional regression term and will thereby extract a full model that minimizes the mean squared error and a sparse model with the minimum number of parameters for the best fit (see Materials and Methods). This class of model is useful for predictor selection and avoids potential overfitting.

We fit the ongoing activity of each male responsive neuron during male-male interactions in time bins of $1 \mathrm{~s}$ with our suite of behavioral predictors and extracted the coefficients for the best sparse model. An example of the activity across the inter-male interaction (from the introduction of the male stimulus to the removal of the male stimulus) is shown in Figure $8 D$ (blue trace) with the model using the best-fit coefficients (in this case, intermale distance and latency to next attack) shown overlaid (red trace). Across the population, we found that the terms with nonzero coefficients most often included by the sparse lasso model and therefore determined to be the most predictive were intermale distance, resident and intruder velocity, latency to attack, and elapsed time (Fig. 8A). We plotted the distribution of coefficients for each parameter identified as a significant predictor in the sparse model and examined whether these distributions were significantly different from zero, indicating that the relationship between the predictor and the activity goes in a consistent direction. We found that the distribution of coefficients for distance, movement, and aggression-related parameters were consistently different from zero (Fig. $8 B$ ). To assess the relative contributions of distance and velocity to $\mathrm{VMHvl}$ activity, we compared the distribution of standardized $\beta$ weights from the model between these parameters. In both the full and sparse model, weights for the distance predictor were significantly higher than weights for the velocity parameter $(p \ll 0.001$ for the full model and for the sparse model, Wilcoxon's signed-rank test, $n=93$ neurons in 5 animals that could be fit with the model).
In many cases, the sparse model predicts a substantial proportion of the variance of ongoing neural activity during the intermale epoch. We plotted the distribution of the $r^{2}$ value for the population of male responsive neurons for the set of predictors that minimized the mean squared error (Fig. $8 C$, white bars) and overlaid the values of $r^{2}$ for the neurons using the sparse model (Fig. 8C, black bars). Using this relatively limited set of predictors, in some cases, we can predict up to half of the total variance in a freely moving animal during a complex social encounter (mean $r^{2}$ value for full model $=0.203, n=93$ male responsive neurons that contained both attack and groom session; mean $r^{2}$ value for sparse model $=0.241, n=58$ neurons).

Does attack latency still have an effect on VMHvl activity after accounting for distance and movement parameters as suggested by the difference map in Figure 7? After using regression with L1 regularization to programmatically identify behavioral parameters most associated with changes in activity during inter-male encounters, we tested the significance of each parameter within a reduced model containing the most frequently identified regression terms: inter-male distance, velocity, elapsed time, and latency to attack. For each neuron, we regressed the activity with these parameters and used an $F$ test to assess the contribution of each parameter against the null distribution. We found that 33 of 93 (35.5\%, significant after FDR of 0.05) of male responsive neurons had significant regression coefficients for the inclusion of the latency to attack, even after accounting for other parameters. These data strongly suggest that, in addition to being sensitive to distance and movement parameters during inter-male encounters, $\mathrm{VMHvl}$ activity is also sensitive to future aggression-related parameters during ongoing behavior.

\section{Discussion}

In examining the relationship between hypothalamic activity and aggression, we found that the responses of VMHvl cells are complex and heterogeneous. Rather than responding exclusively during attack as would be expected for a structure involved in motor initiation, the response in a subpopulation of male responsive cells is dominated by male olfactory input, influenced by attack history, reflective of motor intensity during fighting, and predictive of timing and duration of future attack events. Our data position the role of the VMHvl not as a simple action pattern generator for attack but instead as playing a more complex role in encoding both the proximity to relevant sensory stimuli and the predisposition or motivation to attack. This analytical framework may be more broadly applied to understanding how neural activity in the hypothalamus mediates other motivated behaviors, such as mating and feeding.

\section{Sensory coding in the VMHvl}

We find clear evidence supporting the role of the VMHvl in signaling the presence of male-specific olfactory cues. Male responsive VMHvl neurons show acute increases in activity during investigation of male mouse urine, and activity decays with increasing distance from its source. For rodent social communication, olfaction is the primary signaling mechanism. Dampening olfactory input from either the accessory olfactory system (AOS) or the main olfactory system (MOS) strongly suppresses intermale aggression (Bean, 1982; Mandiyan et al., 2005; Wang et al., 2006). Mice deficient in TrpC2, a putative ion channel expressed exclusively in the mouse vomeronasal organ, display sexual behavior instead of inter-male aggression toward male intruders (Leypold et al., 2002; Stowers et al., 2002). In contrast to olfaction, visual and auditory cues appear to play only minor roles in 
A

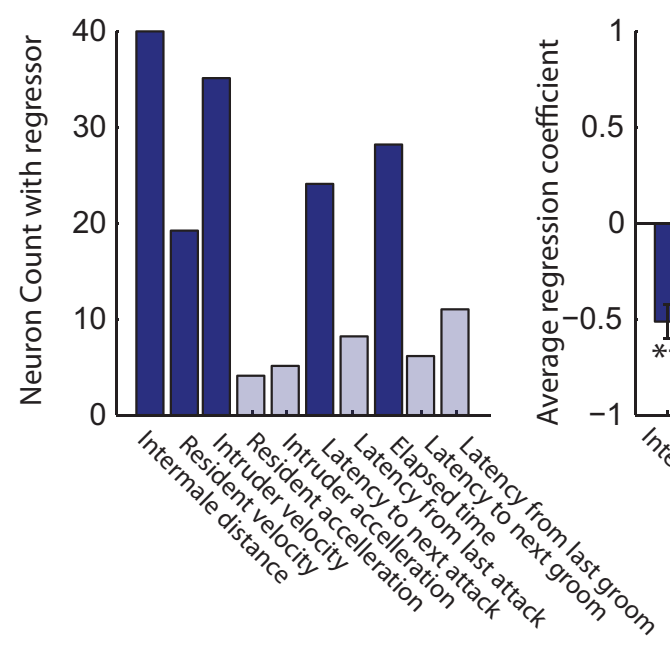

B

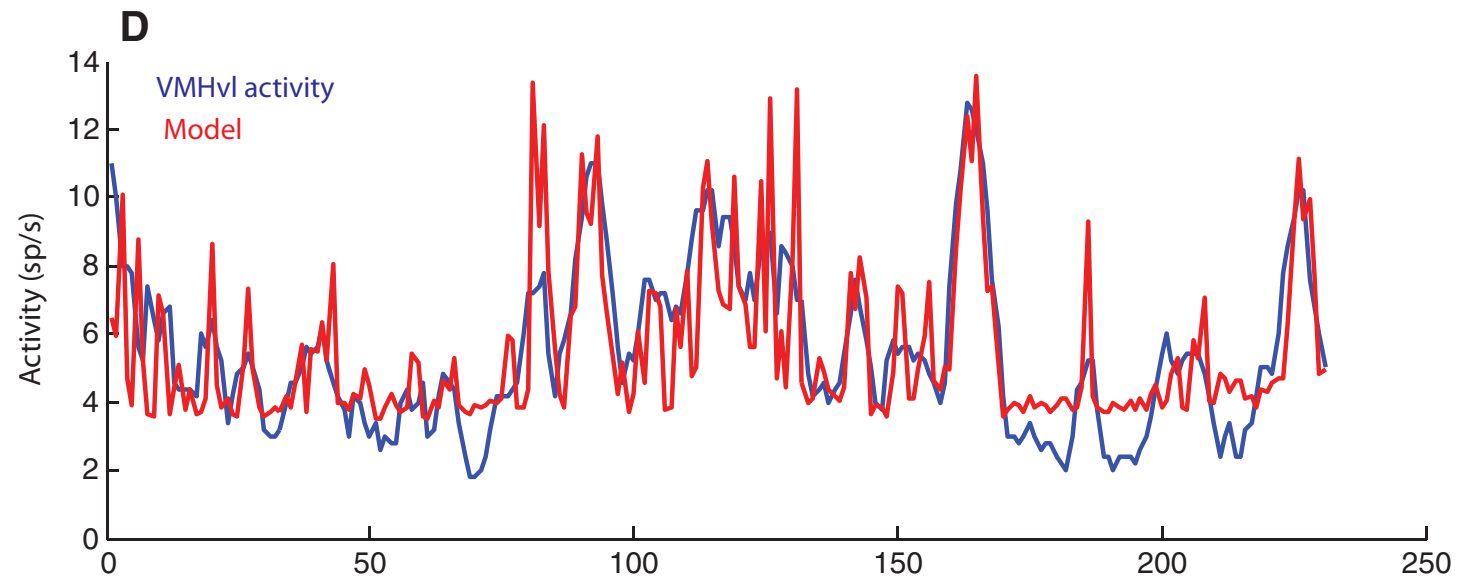

Time during male encounter (s)

Figure 8. Model of VMHvl activity during inter-male interactions with aggression-specific parameters using regularized linear regression. $\boldsymbol{A}$, Number of neurons including each parameter in the sparse model. Neurons were regressed individually, and the $y$-axis indicates the number of neurons that include each parameter as a significant regressor to the model. Darker bars indicate parameters most frequently contributing to the model and used for subsequent model. $\boldsymbol{B}$, Distribution of regression coefficients for parameters identified as contributing to the sparse model $\left({ }^{*} p<\right.$ $0.05,{ }^{* *} p<0.001, t$ test; $n=93$ neurons in 5 animals). C, Variance explained $\left(r^{2}\right)$ by regularized model for each neuron for the full model (with minimized mean squared error, open bars), overlaid with $r^{2}$ values for the sparse model (filled bars). Neurons with all zero coefficients were eliminated from this analysis. $\boldsymbol{D}$, One example showing the fit between the model and cell activity during male stimulus presentation. The example model includes only two parameters: inter-male distance and latency to attack.

aggression. When animals were deprived of visual and auditory inputs, they were less efficient in detecting and keeping track of an intruder, but biting, the defining component of rodent aggression, was primarily unaffected (Hedrich and Bullock, 2004). Here, we observed a small but significant difference in VMHvl cell activity when animals were investigating an anesthetized male compared with an intact male, suggesting that auditory or visual cues associated with the movement of an intruder may also contribute to VMHvl activity in a minor role.

Olfactory inputs may arrive at the VMHvl through two separable pathways. Because activation of AOS typically requires direct nasal contact with sources of pheromones (Wysocki et al., 1980; Luo et al., 2003), increased VMHvl activity in response to male stimuli at a distance is likely attributable to MOS input, whereas AOS input may increase VMHvl activity during close investigation (Leypold et al., 2002; Stowers et al., 2002; Mandiyan et al., 2005; Wang et al., 2006). These signals likely converge on the VMHvl through the medial amygdala and cortical amygdala posterolateral area, which are direct targets of the accessory and main olfactory bulb, respectively (Canteras et al., 1992, 1995).
How then can we reconcile that optogenetic activation of the VMHvl initiates attack (Lin et al., 2011), yet VMHvl cells are highly responsive to male olfactory cues in the absence of an intruder? One possibility is that VMHvl is primarily an olfactory relay and that its artificial activation provides a fictive male conspecific smell. However, our analysis indicates that VMHvl responses carry rich information regarding past, current, and future attacks, suggesting that a purely sensory role for the VMHvl is overly simplified. Furthermore, optogenetic activation of the VMHvl can sometimes direct attack toward a nonsocial target (e.g., an inflated rubber glove) that will not be a natural target of attack even if supplemented with male conspecific odorant (Lin et al., 2011), suggesting that the odor cue alone is not sufficient to drive attack.

A second possibility is that the VMHvl performs a sensorimotor transformation, a process by which incoming sensory information is converted into aggression-specific motor commands. This hypothesis is supported by our observation that activity in male responsive VMHvl neurons encodes not only proximity to relevant sensory stimuli but also is correlated with attack velocity. 
We also observed that some neurons respond primarily during attack, some only during investigation, and others during both behaviors. These three classes of neurons could in principle implement a sensorimotor transformation in which the attackselective neurons represent the final output layer for motor control. However, the results of stimulation experiments suggest that activation of the $\mathrm{VMHvl}$ does not activate a fixed motor plan. In the absence of a suitable attack target, $\mathrm{VMHvl}$ stimulation does not change an animal's behavior overtly, demonstrating that social context is critical for evoked aggression (Lin et al., 2011). Furthermore, optogenetic stimulation-induced attack from the VMHvl may occur after long delays ( $>1 \mathrm{~min}$ ) even when a target for attack is readily available (our unpublished results).

A third possibility is that VMHvl performs a sensorimotivation transformation, a process by which sensory stimuli are converted into activity that represents a specific motivational state. Changes in motivational state attributable to increased input from relevant sensory stimuli may increase the likelihood of a suite of actions related to aggression, including but not limited to attack. Downstream neurons may function by slowly accumulating spikes arriving from VMHvl to a floating threshold, the height of which may determine certain aspects of upcoming attack behavior, such as the duration of attack.

\section{Aggressive motivation and attack prediction signals in the VMHvl}

Although the precise definition remains controversial, the term motivation is often used to describe the arousal state that drives an organism to action toward a desired goal. Here we use attack latency to quantify the ongoing level of aggressive motivation and examine its relationship to VMHvl activity. We find that VMHvl activity is negatively correlated with the latency to the next attack, i.e., activity is increased when attack latency is shorter. In a subset of neurons, these effects of attack are significant even after controlling for fluctuations in inter-male distance and velocities of the resident and intruder. In addition, we found that increased VMHvl activity during investigation predicts whether that behavior will lead to attack as opposed to a non-aggressive behavior and that activity leading up to the onset of an attack predicts the duration of the next attack. Furthermore, we found that VMHvl activity during attack is strongly correlated with the amount of time elapsed since the last attack, in which longer elapsed time is associated with increased VMHvl attack response. These data are consistent with the motivational theory proposed by Tinbergen (1951) who suggested that motivation can accumulate between consummatory actions. In our data, increased motivation over time is reflected by both the neural activity and the mouse's behavior: after long inter-attack intervals, attack activity is high and the duration of the subsequent attack is long.

Exposure to aggression-relevant sensory cues may also increase aggressive motivation. The fact that VMHvl neurons can be activated by a purely olfactory cue, male urine, does not exclude its potential role in motivation, because those cues can promote such internal states (Archer, 1968; Mugford, 1973). Neural activity underlying motivation may outlast the presence of acute sensory stimuli or the cessation of action. Consistent with this prediction, we observed that, after exposure to male stimulus, the firing rates of individual VMHvl neurons remained elevated. Although this apparent "persistent" activity could, in the case of intact or anesthetized males, be attributable to residual bodily deposits, this seems much less likely in the case of male urine that was introduced in a plastic dish.

The origin of a sensory-independent or persistent motiva- tional signal is less clear. Activity could be generated within the hypothalamus by feedback excitation, by feedforward inputs, or by the effects of neuromodulators. Neurons in the VMHvl are primarily glutamatergic and form dense intranuclear connections. This architecture could potentially support recurrent excitation to maintain or amplify a sensory signal from afferent inputs (Millhouse, 1973; Nishizuka and Pfaff, 1989; Canteras et al., 1994). Neurons in the VMHvl also express a variety of neuromodulator receptors, including serotonin $1 \mathrm{~A}, 2 \mathrm{~A}$, and $2 \mathrm{C}$, muscarinic acetylcholine, and oxytocin receptors (Freund-Mercier et al., 1987; Levey, 1993; Zhang et al., 1999; Huang et al., 2004). Because many neuromodulators, such as serotonin and dopamine, change their levels dynamically during the course of aggressive behaviors, they may potentiate VMHvl cell excitability under conditions of high aggressive arousal state or during the post-male stimulus period.

In these experiments, we only assess changes in "withinanimal" motivation, using detailed recordings performed in a relatively small number of animals. These methods do not take into account potential variability between animals during aggression and do not preclude the involvement of hypothalamic substrates in individual differences in motivation. In studies of other motivated behaviors, such as feeding, operant conditioning procedures such as bar pressing or nose poking are often used to quantitatively measure animals' motivation and assess the amount of work the animal is willing to do to obtain access to resources or rewards. Previous studies showed that the opportunity to attack a weaker intruder may potentially reinforce operant conditioning (Fish et al., 2002; May and Kennedy, 2009). These data suggest that manipulating activity in the VMHvl may affect animals' performance on such tasks and will be a goal of future research.

\section{References}

Archer J (1968) The effect of strange male odor on aggressive behavior in male mice. J Mammal 49:572-575. CrossRef Medline

Bard P (1928) A diencephalic mechanism for the expression of rage with special reference to the sympathetic nervous system. Am J Physiol 84: $490-515$.

Bean NJ (1982) Modulation of agonistic behavior by the dual olfactory system in male mice. Physiol Behav 29:433-437. CrossRef Medline

Bruce HM (1965) Effect of castration on the reproductive pheromones of male mice. J Reprod Fertil 10:141-143. CrossRef Medline

Canteras NS, Simerly RB, Swanson LW (1992) Connections of the posterior nucleus of the amygdala. J Comp Neurol 324:143-179. CrossRef Medline

Canteras NS, Simerly RB, Swanson LW (1994) Organization of projections from the ventromedial nucleus of the hypothalamus: a Phaseolus vulgaris-leucoagglutinin study in the rat. J Comp Neurol 348:41-79. CrossRef Medline

Canteras NS, Simerly RB, Swanson LW (1995) Organization of projections from the medial nucleus of the amygdala: a PHAL study in the rat. J Comp Neurol 360:213-245. CrossRef Medline

Dollar P, Welinder P, Perona P (2010) Cascaded pose regression. Paper presented at the 23rd IEEE Conference on Computer Vision and Pattern Recognition. San Francisco, June 13-18, 2010.

Ettrup KS, Sørensen JC, Rodell A, Alstrup AK, Bjarkam CR (2012) Hypothalamic deep brain stimulation influences autonomic and limbic circuitry involved in the regulation of aggression and cardiocerebrovascular control in the Gottingen minipig. Stereotact Funct Neurosurg 90:281291. CrossRef Medline

Fish EW, De Bold JF, Miczek KA (2002) Aggressive behavior as a reinforcer in mice: activation by allopregnanolone. Psychopharmacology 163:459466. CrossRef Medline

Freund-Mercier MJ, Stoeckel ME, Palacios JM, Pazos A, Reichhart JM, Porte A, Richard P (1987) Pharmacological characteristics and anatomical distribution of $\left[{ }^{3} \mathrm{H}\right]$ oxytocin-binding sites in the Wistar rat brain studied by autoradiography. Neuroscience 20:599-614. CrossRef Medline 
Hedrich HJ, Bullock GR (2004) The laboratory mouse. Amsterdam: Elsevier Academic.

Huang XF, Han M, Storlien LH (2004) Differential expression of 5-HT2A and 5-HT2c receptor mRNAs in mice prone, or resistant, to chronic high-fat diet-induced obesity. Mol Brain Res 127:39-47. CrossRef Medline

Kruk MR, Van der Poel AM, Meelis W, Hermans J, Mostert PG, Mos J, Lohman AHM (1983) Discriminant-analysis of the localization of aggression-inducing electrode placements in the hypothalamus of male rats. Brain Res 260:61-79. CrossRef Medline

Kruk MR, Halász J, Meelis W, Haller J (2004) Fast positive feedback between the adrenocortical stress response and a brain mechanism involved in aggressive behavior. Behav Neurosci 118:1062-1070. CrossRef Medline

Levey AI (1993) Immunological localization of M1-M5 muscarinic acetylcholine receptors in peripheral tissues and brain. Life Sci 52:441-448. CrossRef Medline

Leypold BG, Yu CR, Leinders-Zufall T, Kim MM, Zufall F, Axel R (2002) Altered sexual and social behaviors in trp2 mutant mice. Proc Natl Acad Sci U S A 99:6376-6381. CrossRef Medline

Lin D, Boyle MP, Dollar P, Lee H, Lein ES, Perona P, Anderson DJ (2011) Functional identification of an aggression locus in the mouse hypothalamus. Nature 470:221-226. CrossRef Medline

Lipp HP, Hunsperger RW (1978) Threat, attack and flight elicited by electrical stimulation of the ventromedial hypothalamus of the marmoset monkey Callithrix jacchus. Brain Behav Evol 15:260-293. CrossRef Medline

Luo M, Fee MS, Katz LC (2003) Encoding pheromonal signals in the accessory olfactory bulb of behaving mice. Science 299:1196-1201. CrossRef Medline

Mandiyan VS, Coats JK, Shah NM (2005) Deficits in sexual and aggressive behaviors in Cnga2 mutant mice. Nat Neurosci 8:1660-1662. CrossRef Medline

May ME, Kennedy CH (2009) Aggression as positive reinforcement in mice under various ratio- and time-based reinforcement schedules. J Exp Anal Behav 91:185-196. CrossRef Medline
Millhouse OE (1973) The organization of the ventromedial hypothalamic nucleus. Brain Res 55:71-87. CrossRef Medline

Mugford RA (1973) Intermale fighting affected by home-cage odors of male and female mice. J Comp Physiol Psychol 84:289-295. CrossRef Medline

Nishizuka M, Pfaff DW (1989) Intrinsic synapses in the ventromedial nucleus of the hypothalamus: an ultrastructural study. J Comp Neurol 286: 260-268. CrossRef Medline

Novotny M, Harvey S, Jemiolo B, Alberts J (1985) Synthetic pheromones that promote inter-male aggression in mice. Proc Natl Acad Sci U S A 82:2059-2061. CrossRef Medline

Putkonen PT (1966) Attack elicited by forebrain and hypothalamic stimulation in the chicken. Experientia 22:405-407. CrossRef Medline

Roberts WW, Kiess HO (1964) Motivational properties of hypothalamic aggression in cats. J Comp Physiol Psych 58:187-193.

Siegel A, Pott CB (1988) Neural substrates of aggression and flight in the cat. Prog Neurobiol 31:261-283. CrossRef Medline

Siegel A, Roeling TAP, Gregg TR, Kruk MR (1999) Neuropharmacology of brain-stimulation-evoked aggression. Neurosci Biobehav Res 23:359_ 389. CrossRef Medline

Stowers L, Holy TE, Meister M, Dulac C, Koentges G (2002) Loss of sex discrimination and male-male aggression in mice deficient for TRP2. Science 295:1493-1500. CrossRef Medline

Tibshirani R (1996) Regression shrinkage and selection via the Lasso. J R Stat Soc B Met 58:267-288.

Tinbergen N (1951) The study of instinct. Oxford, UK: Clarendon.

Wang Z, Balet Sindreu C, Li V, Nudelman A, Chan GCK, Storm DR (2006) Pheromone detection in male mice depends on signaling through the type 3 adenylyl cyclase in the main olfactory epithelium. J Neurosci 26:73757379. CrossRef Medline

Wysocki CJ, Wellington JL, Beauchamp GK (1980) Access of urinary nonvolatiles to the mammalian vomeronasal organ. Science 207:781-783. CrossRef Medline

Zhang L, Ma W, Barker JL, Rubinow DR (1999) Sex differences in expression of serotonin receptors (subtypes 1A and $2 \mathrm{~A}$ ) in rat brain: a possible role of testosterone. Neuroscience 94:251-259. CrossRef Medline 